\title{
The Application of Minimalist Style in Interior Design
}

\author{
Yingfeng Kuang \\ South China university of Technology \\ Guangzhou, China 510000
}

\author{
Weihai Zhang* \\ South China university of Technology \\ Guangzhou, China 510000 \\ *Corresponding author
}

\begin{abstract}
Minimalist style first appeared in the field of painting and sculpture; it emphasized using extremely simple handling to express rich and profound artistic language. After years development, it connotation was continuously improved, and gradually became a philosophy thought. Its guiding significance was not only embodied in the original field, but also had a great impact in clothing, culture, industrial design, visual and other fields. The energy crisis that emerged in the 1990s gave it a new extension of the value of energy conservation and environmental protection. As a value in line with the trend of social environment, minimalist in life has been widely accepted. When the interior design is influenced by minimalist, it formed a very unique modern style minimalist style. Minimalist style inherited the minimalist accept "less is more", and followed this principle in the material, color and light. The minimalist style developed most is in Germany, Northern Europe and Japan, Germany's Bauhaus proposed that design should follow "Less is more" in form and take functional service objects as its core. The form of design in Northern Europe is simple, which shows the enjoyment and enthusiasm to life. That in Japan values practical functions with its unique characteristics. In this paper, the author shows the minimalist style concretely by analyzing the "Farnsworth House" "Nordic minimalist villa" and describes the requirements of minimalist style in the form, material, color and light. He follows the principle that the form serves for the function to save space and displays the state of minimalist way and its style.
\end{abstract} style

Keywords-minimalism, life style; practicability; minimalist

\section{INTRODUCTION}

In the wave of rapid socio-economic development, the rapid pace of urban life is inevitable, so the pressure of learning and work faced by people will be more and more. Therefore, some people choose to release the mood in the singing, some people choose to go on a trip and some people choose to forget the trouble by reading and so on. However, these releasing ways are temporary and more people have understood great burden both on body and mental because they cannot excrete these pressures for various reasons for a long time. How to make people relief of stress effectively and sustainably has become an important issue in the current society. According to the survey, in the spare time, the place people stayed longest is home, so releasing people's mental pressure in the residential place is the most suitable way. As a vital part of people's living environment, the design of residential environment draws more and more people's attention. How to demonstrate the various functions of the house and meet the user's living needs and spiritual needs should be considered by the interior designers deeply.

The improvement of material conditions and the openness of thinking make people think about things not only on the surface, but more and more on a deeper level of spiritual and quality, they require more and more on 1 living environment. Because of pressures of busy life, learning and work, people are increasingly eager to release them in the spare time to enjoy life. They forget the busy, complicated, tired reality temporarily to comply with the heart and enjoy peace. In the early stages of economic development, people are busy working. If you ask others why are they so busy, you will certainly be considered to be dreamless and slothful. After the reform and opening up, people began to reflect and wanted to change their life. Under the enormous social pressure, more and more people want to enjoy a simple and tasteful life.

\section{THE CONCEPT AND DEVELOPMENT OF MinIMALISM}

\section{A. Historical Origin and Concepts of Minimalism}

Minimalism officially appeared in 1950s and 1960s, it is the extension and development of minimingism. Minimalism first appeared in the field of painting and sculpture, it emphasized that the most important expression of the painting is the relationship between form and color, advocated to express the artistic language with simple outer contours and colors and simplify to the extreme. In 1960s, as a special form of art for the reactionary to the prevailing formalism, it undergone a preliminary formation and development, and by 1980s, it became an influential philosophy thought in Europe and the United States. As a kind of concept of removal of complex and back to the nature, it spread to the visual, music, literature and many other areas. The energy crisis in the 1990s made people begin to reflect on the commercialization and luxury development of society, pay attention to the importance of the ecological environment and natural resources to the 
development of human beings, while the values of energy conservation and environmental protection cater to the idea of minimalism well. The great pressure of urbanization and the huge load of the environment make people are more eager to be free and comfortable, fresh and simple, which makes minimalism develop rapidly. Minimalism shows a simple and pure framework, brings the viewer into the thought of contemplation by very little decoration, so that it shows the space for thinking without limitation, triggering a complex and exaggerated sense of style. In this way, minimalist becomes a popular trend and a life style in people's lives.

The philosophical thought contained in the minimalism is also reflected in the traditional culture of our country. In the metaphysics, there are many sayings such as "doing nothing while doing everything" "the less is the gain, while the more is the temptation" "to be extremely concentrated with an empty mind" and so on, all of which emphasize following the nature and adhering to the essence; it is also referred in Zen that "turning complication to simplification" and "all is empty", that is to say restoring the nature of the material, abandoning all the fetters of the soul and returning to the beginning. This is also consistent with the simpleness and brief modification advocated by minimalism.

Minimalism, is also known as minimalist or minimalist art. As a reactionary to concrete expression, it develops to the acme and shows itself to viewers with the original material or form. The designers reduce form maximally in order to pursuing simpleness to the best and giving a strong sensory feel, so as to obtain the essence of material and to show the most wonderful feelings with the most appropriate and least way. The minimalist advocates "less is more". Less is not as simple as possible, but is to abandon all the factors that interfere with the subject, and reduce the formal elements to a minimum to express the profound and rich meaning with a simple element and achieve a simple and free form and an elegant taste, which is a high-quality embodiment. As a philosophical thought, minimalism involves many areas of life, and the specific requirements are different in different areas. Its core idea is to pursue the most simple and clear form of expression to express the elegant taste.

\section{B. The Life Style of Minimalist}

\section{We all have extremely same experiences!}

From the birth, what we bring to the world is just our bodies, but with the time going by, there are many things craved on us. The first cloth, toy, our own room, the first book, cup and bicycle and so on. We have more and more things, so we have to tidy more and more. We are unwilling to abandon the used toys, we gain various prizes and certificates and we buy kinds of souvenir, etc. The space for storing becomes larger and larger. For many times we are tired of tiding these things, and gathering up to desert them, but we don't do that at last. This troubles us time and again, wastes our energy and time once and again, however, have we considered that these things left in fact are of no use, only to make the home like a shelter, both accounting for space and increasing the pressure of finishing? Have we thought that we should change the state and cut off things that we do not need, give up too much waste, and get rid of the attachment of the items to enjoy life?

When we wake up in the morning, the spacious bedroom makes us pleasant. Eye-drawing and simple furniture seems to make the whole day easy. There aren't many clothes but we like every of them, so we needn't to choose which to wear. The comfortable indoor space enables us to enjoy freedom. When getting out, we just need to take the bad containing some things necessary, as light as taking nothing. We do thing we want to do over a cup of coffee. On a sunny weekend, we can go on an outing with some friends, and enjoy the favorite cake in the evening. We can read a few pages of books and have a rest to release our mood.

Try to live extremely simple, to understand your original will and real need without the interference of external factors and devote your energy and time to what you really need. To read books you like indeed, travel to the places you want to go and attend your favorite class of interest to learn and improve yourself. To restrain your motivation to first-sight goods and buy the best one if you need, use it to the full extent and apply the environment protection to your daily behaviors only for the inner peace. To get rid of useless information, look through micro blogs or details seldom to focus on the useful things. You will find there is much spare time by doing this. We can do things timely and effectively, and sort out what is unnecessary to keep the quality of life and the state of heart. Attending helpless social activities little and doing more exercises to enjoy life and freedom.

\section{The Application of Minimalist in Other Fields}

The idea "less is more" of minimalist makes its form brief and simple, and gives it a sense of modernization. Under this core, minimalist has a great influence on the development of many fields. It is used in book binding design and shows information fully. Compared with other rich and classical design style, it removes complicated decoration and displays the logical language of the designers and authors in all respects. "Simplicity is promotion". Combining the logic and concept of the designers with the design and showing to the viewers in this unique way gives them different feelings both on sight and heart.

Minimalist style furniture first appeared in the German Bauhaus Institute. It combines rational structure and simple form effectively and adopts green new materials and excellent technology to achieve maximum comfort with minimal cost. The people-oriented concept of minimalist style furniture determines that its shape is the most ergonomic and allows the user to achieve maximum comfort and relaxation; in terms of form, it values design fashion, art form and design language. In the material, it pays more attention to the new green and sustainable materials. Whether it is a chair with unique shape or a sofa stressed the comfort, they can combine function and decoration properly.

Designers of minimalist clothes pay more attention to the comfort and fabric of the materials, as to the pattern, they prefer to the simpleness and line sense and cut fit. They 
value integrity of the fabric and cut as little as possible. They take original texture of the fabric as a visual point, highlight the quality and emphasize the external rationality and quality as well as inner refinement and elegance. On the visual feeling, they mainly select neutral color like black and white because it seems more qualified and fashionable together with other colors. Designers use a simple square or threedimensional geometry as the basis and divide them equally or superimpose repeatedly. They take subtraction as the main principle to shape the appearance and decorate little because they express the fashion, quality and sophistication by the material texture and color.

\section{THE APPLICATION OF MiNIMALISM IN INTERIOR DESIGN}

\section{A. Concept of Minimalist}

Minimalist style is the product after the application of minimalist in the interior design, it inherits minimalist concept "less is more" "return to the original", so it minimizes the violence in the emergence of works and deletes methods, means and content of expression of art maximally to make a strong visual experience and obtain the essence of art. So minimalist style is to remove the extra decoration and pursue the most essential part by the most basic means of expression. So it is simple and neat in sense and more elegant in taste and thinking.

\section{B. The Characteristics of Minimalist Style}

The essence of minimalism can be embodied in its essential concept, which is basically summed up in three respects, namely form, function and material. It is greatly simplified in form, and takes the function as the core of design. It is no longer the design just for form because it stresses "less is more". In addition to the simplification of decorative elements, the form of furniture also has characteristics. Designers use the geometric dot, line and plane in their design, shape the corner with a hard line, simple and clear. The color is also simple in the view, most of which is gray. They value practical and scientific function, comfortable use and convenient and scientific construction. The material should be environmental-friendly, safe and economic.

\section{Research on the Aesthetics and Philosophy of Minimalist Style}

The "less is more" concept of minimalist style, decides that it is not complex in expression. There are no extra decorative elements, but it emphasizes on the design of the visual integrity and formal simplification, its ideal state is to achieve infinite possibility by a simple form of expression. The traditional art of our country - wash painting, it embodies artistic beauty and harmonious beauty in aesthetic thought. The painter just draws a few times with a brush pen, while what is on paper is like floating clouds and flowing water, which is wonderful. This is similar to the original intention of minimalist, that is, to cause infinite subjective feelings with a limited object. In the minimalist color, it also reflects the beauty of harmony. The proper application of black and white gives the effect of color a sense of taste. It seems that they create a painting with black and white in view.

We should learn to use a few things to live a meaningful life. It is mentioned in "Lao Tzu" that "people should live according to the earth, and the earth moves according to the heaven, while the heaven runs according to the principle, and the principle is formed according to the nature". It refers that all things are in accordance with certain laws, and something comes from nothing and ends in nothing, which is the starting point and the end point of all things as well as the final basis for change and motion. There is a saying in metaphysics that "the less gained while the more confused". It means people can understand the heart, acquire the fun and freedom of spirit in a simple environment while the surrounding complex things make people confused. Confucianism requires that people should have a way of thinking that transcends material desires for moral values and ideals and beliefs. The thought in Zen "to be empty, ethereal, simple and far-sighted ", "to be bright and clean, to be vivid" means to get rid of the shackles of foreign objects, restore the original material and get the inner peace.

\section{The Green Initiative and Sustainable Concept of Minimalist Style}

The green initiative of minimalist style is mainly reflected in the application of materials. In the materials of furniture, they don't use fur although it represents elegance, noble and quality. This is because the fur comes from animals, which is unacceptable in minimalist. Therefore, the materials of indoor furniture are mainly cotton fabric and carbon fiber, transparent environmental-friendly. Fabric's texture feels comfortable at people's first sight. Furniture materials are close to nature, mostly are wood and other natural materials. The basic paint on the wall is latex paint, which is easy to paint and simple in shape and reduce many troubles when decorating a second time. "Less is more", is to simplify the elements in the entire interior space and the materials used in the decoration will be greatly reduced.

\section{E. Analysis on the Farnsworth House and Nordic Minimalist Style Villa}

Farnsworth House is a representative work of the modernist architect Ludwig Mies van der Rohe, it is specially designed for his favorite female doctor, Edith Farnsworth. In this works, he plays the reducing spirit to the full extent. The Farnsworth is a separate house in a jungle. It looks like a white, exquisite box from the outside, with white lacquered steel as the structural frame of the whole house. All the walls are made of glass, glass and the proportion of the size is also strictly in line with gold section scale. The steel and glass texture and hard lines make the entire design full of modern atmosphere. The permeability of glass replaces the obstructed wall, connecting indoor and outdoor space together visually. It reflects the extremely minimalist ideas. Steel lines and glass plane, contrast between block steel and transparent glass, show the ingenious application in materials. 
But such a beautiful work is not what the female doctor like because the permeability of glass couldn't provide a private life for her. It cannot work as original intention making the owner enjoy the freedom and transparency. What's more, the glass cannot maintain and evacuate the temperature like solid wall or block light. "Fig. 1"

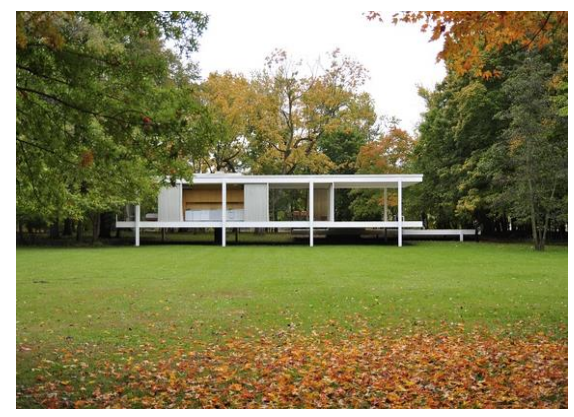

Fig. 1. Farnsworth house.

This simple design of the house also conforms to the minimalist style. It is mainly embodied by texture of materials, color match and the selection of furniture and so on. All the walls are painted white, weakening the division of space, giving a relaxed feeling to people. The variety of light coming into entire space is very soft. The furniture is mainly black and white, going match with walls. The contrast of colors makes people feel good. The cloth is mostly cotton and linen fabrics, its soft and comfortable feeling gives people an impulse to relax. The ground is decorated with wood flooring with parts carpet. The color is slightly obsolete, which is more nostalgic, increasing design quality. Some parts of the wood are painted white to decorate walls, which enriches the vision without complex feelings. A small part is decorated with bright colors, making the design closer to life and vivid. However, the design and decorative elements are relatively complicated, weakening the minimalist theme. "Fig. 1", "Fig. 2"
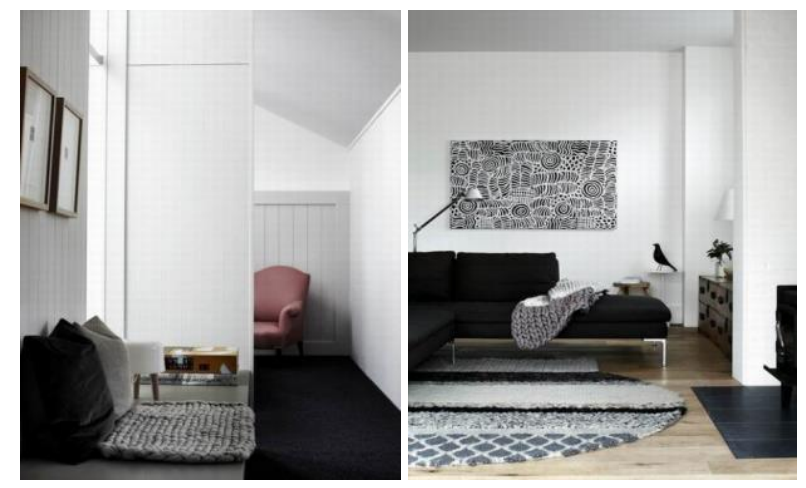

Fig. 2. Scandinavian minimalist villa design

\section{DESIGN METHODS OF THE MinimALIST INTERIOR DESIGN}

\section{A. Proper Division of the Living Space}

Interior design is people-oriented and the division of space and function serves for use to achieve maximum value with the limited space. The interior design is to coordinate the relationship among life, spirit and the surrounding environment. To analysis space with the artistic view and to improve the design with technical means and create, promote, organize indoor space based on human life and spiritual needs. Before the design, the area of each space will be measured, so that the designers will design and divide the functional area according to the indoor space. Whether the size of the area is appropriate depends on the furniture layout and ergonomics. For example, when putting sofa into living room, the distance between the sofa and the TV wall is suitable for people to walk; when laying a table, the restaurant space can cover other soft equipment or not. That is, the division of the function should be rigorous. Designers should consider it is practical and convenient after design. The use of space should be reasonable, under the principle of keeping unity to reduce the waste of space.

\section{B. The Selection of Materials and Colors}

Minimalist style interior design is the product of widely application of minimalist life, so the choice of materials is also affected by minimalism. It advocates using proper, reasonable and materials with geographical characteristics. To be green initiative, there are many kinds of materials to be chosen from such as brick, steel, latex paint and other common stencil profiles. In order to highlight the theme and increase visual effects, designers may just use a single material in a process. The wall is an important place to highlight the style, so the wall also has an important role in reflecting a variety of artificial light and natural light. The material of the wall makes the indoor light softer after light refraction and diffuse reflection. Fabrics are mainly cotton and linen without too much obvious texture in order to maintain original material's touch feeling and visual sense and highlight simplicity and other different taste.

The colors are mostly black and white, so what is the most important is to coordinate the proportion of black and white with the main color proportion as its core, matching with a few bright colors or natural colors to make the entire space more vibrant and layered.

\section{The Selection of Furniture}

Modern minimalist furniture is comfortable in function and simple in appearance, it can be said to be geometric. The concept of furniture design first appeared in the German Bauhaus Design Institute, its development is the most typical now and the formation of a culture is in Japan. Its idea is similar to zazen in Buddhist. It combines culture precipitation with modern form, making people feel simple but extraordinary. They use kinds of materials like fiber, fabric, metal, plastic, wood and so on. In shape, designers adopt lines and basic geometry. This conforms to the reduction principle and is consistent with the composition of point line theory, avoiding cumbersome processes. In order to increase the indoor space elements and avoid a single material in large area, as an important element of indoor composition, furniture should be placed in line with the whole environment and match the surrounding environment. The color also complies with the minimalist framework, so 
they are mainly black and white, simple with connotation. Rarely appeared dotted elements make the design pure and generous. All of these give the users a sense of relaxing, comfort and freedom.

\section{The Application of Light}

Light is very important in the indoor environment, whether it is natural light or artificial light, if the indoor light is too strong or too weak, the design will be greatly different in terms of beauty. The light shows the sense of the layers of indoor elements and makes the visual sense wonderful. Designers of minimalist style attach great importance to external ambient light. Comparing to the fixed artificial light, designers prefer the freedom and infinite changes of natural light. Different time, different weather, different seasons, convey different languages from the outside and bring people different experiences. Because the short-term changes of night and natural light are not obvious, the artificial light is indispensable. But the hue of artificial light should not be too high, so the white or light color is appropriate. Via the combination of artificial light and ambient light, they will influence mutually, so that the indoor space will be bright and transparent. In terms of lamps, they abandon complex lamps and would like simple spotlights.

\section{CONCLUSION}

After the research on design of minimalist style, the author has a deeper understanding to interior design. All things are not isolated and related to the surrounding things. So does the interior environment. All the rooms in this space seem to be separated, but under the theme of the room, they have their own characteristics without deviating from the integrity. The fun of interior design is that there are infinite possibilities under the same framework. On the premise of customer subjectivity, the designer changes the original things of no connotation to a new one of great quality by their own creation, which is full of visual sense. Via this minimalist interior design, I refreshed my cognitive to the design once again, I hope that this design can make more people realize and understand the application of minimalist style in the indoor design.

\section{REFERENCES}

[1] Li Li. Thinking flyleaf_- the watcher of minimalist arts. [J]. Hwa Chang.2014,15:P91-P91.

[2] Xu Lianming. Taste of Fashion: Analysis of China's Youth Fashion Culture [J]. Academic Journal of Zhongzhou.2013:,01:P72-P76.

[3] Lin Zhen, Wu Yun. From minimalism to new Chinese style [J]. Literature Life(Trimonthly Publication). 2009,03:P85-P86.

[4] Du Guanquan. A brief analysis on the design of indoor minimalist style [J]. Urban construction theory research. 2012,17:P47-P48.

[5] Liao Xuefeng. An Analysis of Minimalist Design from the Sociological Perspective [D].2013. 